\title{
HOW THE LAWS OF PHYSICS CAN BE CONFRONTED WITH EXPERIENCE
}

\begin{abstract}
Nancy Cartwright's arguments in favour of the phenomenological laws and against the fundamental ones are discussed. I support and strengthen her criticism of the standard coveringlaw account but I am sceptical in respect to her radical conclusions that the laws of physics lie. Arguments in favour of the opposite standpoint are based on V. Stjopin's analysis of scientific-theory structure. A theory-change model presented here demonstrates how the fundamental laws of physics can be confronted with experience. Its case studies include the Lorentz-Einstein-Bohr transition and modern General Relativity and Quantum Field Theory unification.
\end{abstract}

\section{INTRODUCTION}

In her brilliant essay-collection How the Laws of Physics Lie Nancy Cartwright develops an original and thought-provoking point of view according to which the theoretical laws of physics are false and inaccurate in contrast to the phenomenological ones. The metaphysical picture that underlies her essays is an Aristotelian belief in the richness and variety of the concrete and particular. Cartwright's distinction between theoretical and phenomenological separates laws which are fundamental and explanatory from those that merely describe. Yet they are the theoretical laws but not the theoretical entities that are false. Causal reasoning provides good grounds for our belief in theoretical entities. The propositions to which we commit ourselves when we accept a causal explanation are highly detailed causal principles and concrete phenomenological laws, not the abstract equations of a fundamental theory.

Cartwright's paradoxical standpoint is defended with three different but interrelated arguments:

(1) The explanatory power of fundamental laws does not argue for their truth.

(2) The way they are used in explanation argues for their falsehood. We explain by ceteris paribus laws, by composition of causes, and by approximations that improve on what the fundamental laws dictate. In all of these cases the fundamental laws do not get the facts right. 
(3) The appearance of truth comes from a bad model of explanation.

Though Cartwright's arguments against the standard covering-law account seem to me very convincing, her anti-realist conclusions seem to be too radical due to following reasons:

(i) Her analysis of fundamental-theory structure and functioning misses some important aspects. Her case studies are limited by the quantum-theory domain.

(ii) The emphasis is laid on explanations but not predictions. However, they are predictions that usually constitute the strongest argument in favour of the fundamental theory validity. (T. Young's experiment, Mercury perihelion precession, $2,7^{\circ} \mathrm{K}$ background radiation, etc.).

(iii) In contrast to the static aspect, Cartwright's study almost completely lacks the dynamical one. The processes of fundamental-laws genesis and refutation are ignored. The Kuhnian anthropologist arguments seem to tell us that dynamics belongs to external history and is due to socio-cultural factors. Cartwright's results are in excellent accord with the so-called ,strong programme" in the sociology of science (S. Barnes, D. Bloor et al.) since she considers the fundamental laws as socio-psychological tools for knowledge-organization. I think that this departure from internalist approach is too radical to believe in and too deviating from the opinions of ,silent majority” of natural-science researchers. I doubt that scientists themselves will be prepared to hand over to sociologists of science the task of assessing the validity of the fundamental laws.

My paper aims to take (i) - (iii) into account and to provide an analysis superseding that of N. Cartwright's in respect to aspects described. In the second section I wish to present V. S. Stjopin's analysis of the mature theory structure and functioning and to compare it with that of N. Cartwright's. The third section aims to answer the question „How Can a Fundamental Law of Nature be Refuted?". It proposes a model of theory-change constructed within an internalist approach but capable of adding socio-cultural factors into consideration.

\section{THE FUNDAMENTAL THEORY STRUCTURE AND FUNCTIONING}

According to Stjopin (1976), an abstract theoretical object of a set of abstract theoretical objects of any mature theory belongs either to a subset of basic theoretical objects, or to a subset of derivative theoretical ones. The 
relations between basic objects are described by fundamental laws of the mature theory. The relations between the derivative ones are described by consequences from fundamental laws. For instance, the basic theoretical objects of Maxwellian electrodynamics are „the electric field at a point”, „the magnetic field at a point" and „current density”. Relations between them are described by Maxwell's equations.

The basic theoretical objects of Newtonian mechanics are „the material point”, „the force", „the inertial system of reference". Relations between them are described by Newton's laws. The derivative objects of Newtonian mechanics are „an absolutely rigid body”, „central-symmetric field”, ,a mechanical oscillator”, etc. The relations between them are described by the partial laws of Newtonian mechanics, i.e. by laws of a rigid-body rotation, of movement in central-symmetric field, etc. Basic objects form the basis of a mature theory. It means that each derivative object can join the system of theoretical objects only as a result of constructing basic objects according to certain rules. Basic theoretical objects are constructively independent.

For instance, a derivative object of Newtonian mechanics — the oscillator is constructed from the basis of Newton's theory in the following way. It is assumed that the force which changes the state of motion of the material point is quasi-elastic one. It tends to return the point into an equilibrium state. The system of reference is chosen in which the movement of a material point looks like a periodic one. Thus the derivative object — the oscillator — is constructed as a foundation for derivation of small-amplitudes-oscillations equation. According to this model, we can substitute the quasi-elastic-force expression $F_{x}=k: x$ into the equation $F_{x}=$ $m d^{2} x / d t^{2}$ obtaining instead $m d^{2} x / d t^{2}+k x=0$. Here $\mathrm{x}$ is a delay from an equilibrium state and $k$ is the coefficient of proportionality of respective quasi-elastic force. All derivative objects of a mature theory are organized in subsystems. It means that the propositions of each subtheory should not contradict each other. When introducing a new object, the older ones would not acquire new properties, incompatible with the properties given previously.

In general, the relation of basic subsystem to the derivative ones can be characterized as follows. Each derivative system is obtained from the basis by the process of reduction. It means that a mature theory develops not only due to the formally logical and mathematical tools, but also due to 'Gedanken- experiments' with abstract objects. The reduction is performed by an analysis of the peculiarities of the empirically-fixed domain of reality. This domain can be „looked through" the prism of an ideal model, formed by basic objects's correlations. According to peculiarities of each concrete experimental situation, some constraints are imposed on the basis. It enables us to concretize it, transforming to a subsystem of derivative objects. Then the fundamental equations of the theory are applied to the derivative subsystem. In accordance 
with the peculiarities of the system they are transformed into partial-laws expressions. The informal nature of such procedures converts the inference of each consequence into a special problem-solving operation. The solutions of such problems are included into a mature theory during its genesis. They function as examples of theory applications to concrete cases. Each problem is solved in accordance with primary ,paradigms” (Kuhn, 1962).

So, the abstract objects of a mature theory are organized in a complicated system, that includes the subsystems connected with each other according to the principle of level-hierarchy. The lower-level models are subordinated to the basic one.

To compare the theoretical consequencies with empirical data the derivative system should be transformed into an empirical one. The empirical-scheme constructs differ radically from the derivative theoretical objects. They are not the idealizations now. They can be directly compared with real objects of experiments. Each empirical-scheme objects is compared not with a single empirically-given object, but with a set of such objects instead. The empirical scheme corresponds to a type of empirical situations. For example, the empirical scheme of the experiment with magnetic needle and a conductor corresponds to any experiment with any conductor and any magnetic needle.

Up to this point Stjopin's 1976 analysis of a mature theory structure is obviously identical to that of Cartwright's (1983) with empirical laws instead of phenomenological ones and abstract objects instead of theoretical entities (not to forget mature theory instead of fundamental one). Moreover, Stjopin's methodological scheme, based on the material not only of quantum electrodynamics, but on classical mechanics and classical electrodynamics as well, strengthens arguments against the standard covering-law account. In Stjopin's scheme the process of phenomenological-law derivation appears more complicated and less formal than in Cartwright's since to obtain the empirical laws one has to pass through the intermediate level of abstract-objects organization. Empirical laws are derived from the fundamental ones only with the help of derivative objects. Cartwright's Lamb-shift example obviously fits into this pattern.

Yet Stjopin's analysis possesses some important additional properties crucial to realistic point of view:

a) Each system of derivative objects can be compared with definite set of empirical schemes. Each derivative model is an invariant content of the corresponding class of empirical schemes. The notion ,invariant content” should not be missed with simple induction. It is a familiar fact that it is possible to construct inductive rules which give rise to different generalizations from the same empirical evidence. Each basic system is an invariant content of corresponding derivative models.

b) All the theoretical models - the basic model as well as the derivative ones - reflect not only the object of investigation, but also the properties of corresponding experimental device dealing with that object. Hence each model is 
an ideal scheme of experiments. This made it possible to V. Stjopin to call the fundamental-theory models ,the theoretical schemes". The basic model was called „the Fundamental Theoretical Scheme”, (FTS), and the derivative models were called „the Partial Theoretical Schemes” (PTO). All the theoretical models must be operationally defined. For instance, operational definition of the electric field B of Maxwell's equations should be given not through the measurements with real experimental device. On the contrary, it should be given with a help of the descriptions of relations of an electric field at a point to a test charge. But the abstract objects ,electric field at a point" and ,test charge” are the entities of partial theoretical schemes of Maxwell's theory. And their operational status is determined now by real, and not by ideal measurements. The test charge is determined as such an action of a massive body (charged) on the other when the reciprocal influence of the second body on the first can be ignored.

Thus, the theoretical schemes of fundamental theories have two interrelated aspects: (1) they are models of experimental situations (2) and they are models of objects investigated. They are those two aspects that are crucial to the prediction process. (1) - (2) determine the interaction of the various levels of theoretical and empirical objects' organization. Connection between the levels is a rigid one, and this rigidity lets to introduce correspondingly to a new content of the upper level the objects of the lower level, and even to construct an experimental device for examination of the result predicted.

Now Cartwright's conclusions on the fundamental-laws significance can be opposed. She writes:

The great explanatory and predictive power of our theories lies in their fundamental laws. Nevertheless the content of our scientific knowledge is expressed in the phenomenological laws. (1983:100).

We have already mentioned Stjopin's result according to which the Fundamental Theoretical Scheme is a kind of generalizations of all the partial ones, and the latter are the generalizations of the empirical schemes. From this one can conclude that all the empirical content of the fundamental laws is contained in the empirical ones. But it is wrong. For how can we predict the results of future, not known to us now, unperformed experiments. If we can predict successfully, and our theoretical predictions are confirmed by new experiments, it means that the fundamental-laws content has some novel elements, that cannot be reduced completely to the content of old empirical schemes (or phenomenological laws).

Secondly, Cartwright juxtaposes two types of explanations and favours the causal ones. But her demarcation line between those types is not so distinct as it should be. Just to quote her 1983 book:

Explaining in physics involves two quite different kinds of activities. First, when we explain a phenomenon, we state its causes [...] Second, we fit the phenomenon into a broad theoretical framework which brings together, under one set of fundamental equations, a wide array of different kinds of phenomena [...] The standard covering-law account tries to fit both kinds of explanation into 
the same mould. But the function of the laws is different in the two cases, and so too, I have argued, are their claims to truth. The difference is more than philosophical. We find it in scientific practice (cf. The Reality of Causes in a World of Instrumental Laws). In physics it is usual to give alternative theoretical treatments of the same phenomenon. We construct different models for different purposes, with different equations to describe them. Which is the right model, which the 'true' set of equations? The question is a mistake. One model brings out some aspects of the phenomenon; a different model brings out others. Some equations give a rougher estimate for a quantity of interest, but are easier to solve. No single model serves all purposes best.

Causal explanation is different. We do not tell first one causal story then another, according to our convenience. Maxwell's explanation involving tangential stresses is incompatible with the earlier light pressure account [...] If one of these is adopted, the others are rejected. Alternative causal stories compete in physics in a way in which theoretical treatments do not. Causal stories are treated as if they are true or false, but which theoretical laws ,govern" the phenomenon is a matter of convenience. (Cartwright, 1983: 11).

But what aspect different from Einstein's Special Relativity was brought out by Lorentz's ether theory that explained the same bundle of phenomena: the Michelson-Morley experiment, the experiments of Rayleigh, Descoudre, Roentgen, Lodge, Eihenwald, Trouton, Trouton and Noble, Wilson, Strasser, and some others. Einsteins's and Lorentz's theories were incompatible since the first one denied the existence of ether and the second was based upon it. The number of examples can easily be increased thus washing away Cartwright's demarcation line.

Having considered the fundamental-laws genesis, we must turn to their refutation. The standard covering-law account rejection makes the problem of fundamental-laws confrontation with experience especially difficult and important. If there is no direct and unique way from the fundamental laws to phenomenological ones, how can the fundamental laws be confronted with experience? They are the derivative models that can be directly refuted by experiments, but not the basic one. What are the origins of theory-change in physics? Must we turn to sociology of science, to ,functional" explanations of scientific practice? May be, the methodologist of science should behave as anthropologist who finds

...a people with peculiar custom. The people themselves give several or maybe no reasons for their custom - But the anthropologist conjectures that the custom remains among these people not only for their avowed reasons, but also because other customs or ecological conditions make it very difficult for their society to survive without it. This then is the

„function" of the custom in question, even though it is not practised with any conscious awareness of that function. (Cartwright, 1983:143).

In what follows I aim to describe an internalist model of theory-change, that however admits the influence of external factors.

\section{HOW CAN A FUNDAMENTAL LAW OF PHYSICS BE REFUTED}

Let me start with what I had already considered in the previous Dubrovnik paper (1987). Consider the case when several fundamental theories $T_{1}, T_{2}, T 3, \ldots$ 
with basic models $\mathrm{B}_{1}, \mathrm{~B}_{2}, \mathrm{~B}_{3}, \ldots$ are applied to describe a certain domain of reality. As a result, the systems of cross-bred objects $\mathrm{C}_{1}, \mathrm{C}_{2}, \mathrm{C}_{3}, \ldots$ are constructed. Each cross-system consists of derivative objects constructed from all the $\mathrm{B}_{1}, \mathrm{~B}_{2}, \mathrm{~B}_{3}, \ldots$

Relations between the cross-bred objects are described by $\mathrm{T}_{1}$ statements, as well as by statements of $T_{2}, T_{3}, \ldots$ Hence it is of no wonder that there can appear theoretical objects with incompatible properties resulting from the operation of cross-bred-construction in one of the derivative subsystems of one of the crosstheories. It should give rise to the mutually contradicting statements in all the crosstheories or to „cross-contradictions” (Podgoretzky and Smorodinsky, 1980). To eliminate it, one should think of a global theory $\mathrm{Tg}$, that should comprise all the cross-theories in a certain way.

Two methods of Tg-creation are admissible: reductionist and synthetic. (R) Application of the first one is based on the assumption that one of $T_{1}, T_{2}, T_{3}$ (let it be $\mathrm{Tj}$ ) is ,the true one”, while the others are ,phenomenological theories”. Their basic objects should be constructed from $\mathrm{Bj}$, and their fundamental laws should be derived from $\mathrm{T}_{1}$. (S) Application of the synthetic way must result in new global model $\mathrm{B}_{\mathrm{g}}$, from which all the $\mathrm{B}_{1}, \mathrm{~B}_{2}, \mathrm{~B}_{3}, \ldots$ should be constructed.

Realization of $(\mathrm{R})$ excludes the usage of cross-bred objects. On the contrary, (S) considers them as belonging to the higher (in comparison to $\mathrm{B}_{1}, \mathrm{~B}_{2}, \mathrm{~B}_{3}, \ldots$ ) organization level. Both methods result in creation of scientific research programmes with (R) and (S) presenting their „hard cores”. Each programme is to create its own sequence of scientific theories. In constructing $\mathrm{Tg}$, not theories but research programmes are decisive. A programme must be chosen that can provide the successful resolution of cross-contradiction. I can demonstrate that synthetic programme must, properly realized, provide greater empirically progressive problemshift than a reductionist one.

According to their definition, the global objects are the ones from which the basis's of $T_{1}, T_{2}, T_{3}$ are constructed. The global objects contain information about $\mathrm{B}_{1}, \mathrm{~B}_{2}, \mathrm{~B}_{3}, \ldots$ But the cross-bred objects possess this property also! They

are constructed from all the $\mathrm{B}_{1}, \mathrm{~B}_{2}, \mathrm{~B}_{3}, \ldots$ Moreover. Since the process of a crossbred is construction from the basic ones is possible, the reverse process of reconstructing of basis's from the cross-breeds is possible also. We had already pointed out that it is possible to construct several systems of cross-breds in general. Hence each such system contains only a part of information about their bases. It can reconstruct only some of the $\mathrm{B}_{1}, \mathrm{~B}_{2}, \mathrm{~B}_{3}, \ldots$ properties. Only all the set of various cross-systems possesses all their information about their parents. But this set is always open for new elements, since it is impossible to declair beforehand that all the cross-domains are known to us. Any fundamental theory can suffer the unrestrained development by the partial theories construction form the FTS for each new domain of application. The restrictions can occur only after the cross with the other fundamental theory, but the number of such meetings in unlimited. That is why the global system must occur only as a result of the unification of all the cross- 
bred systems and cannot leave a secluded life. It is always opened for new crossbred systems, and the new elements introduction can endow the old ones by new properties in complete analogy with the object-introduction into cross-bred systems.

The methodological scheme described can be illustrated by the examples from the 19-20 centuries scientific revolution. The modern quantum theory was created by unfication of Bohr's cross-bred atom models, of Einstein's cross-bred semicorpuscular radiation theory, of special relativity, etc. This process is not finished until now. „I think one ought to say that the problem of reconciling quantum theory and relativity is not solved" (Dirac, 1973, p. 10-11).

Coordination of Special Relativity with Newton's theory of gravitation led to General Relativity creation (see at full length Nugayev, 1987). Einstein's efforts to incorporate Newtonian gravity into the SR framework began in 1907 when he had to prepare a review for Jahrbuch der Radioaktivitat. His first effort was unsuccessful since simple-minded generalizations (though Lorentz-invariant) failed to explain anomalous Mercury perihelion motion. General Relativity (GR) creation led to the problem of GR and Quantum Field Theory (QFT) unification. The problem is not solved until now, but the first cross-bred systems are created already within the „quantum field theory in curved spaces” (Birrell, Davies, 1982). S. W. Hawking's analysis of the ,particle creation by black holes" is an obvious example of cross-theory. From its very creation the theory met with paradoxes caused by the lack of agreement between GR and QFT (Fulling, 1973; De Witt, 1976). One of the leading black-hole theorists points out that the paradoxes' cause consists in „semiclassical" black-hole evaporation theory. The gravitational field is treated „classically" (as a spacetime metric) there, while its sources are described in terms of quantum field theory. „The basic equation of $G R, G_{m}=T_{m}$ contains incompatible quantities on the two sides of the equals sign. Furthermore, this incompatibility has consequences even at the present experimental scale" (Unruh, 1980, p. 154). 
The QFT vacuum plays the role of the cross-bred object here. Its regularization methods were elaborated for flat-spacetime cases. They do not work in strong gravitational fields since vacuum possesses there infinite energy owing to its own gravitational field. It seems to me that the vacuum took place already of a global theoretical object in modern superunification theories (Chyba, 1985), in inflation theories that predict some interesting effects as consequencies of spontaneous symmetry-breaking. It is important that here the synthetic global theory creation seems to follow the lines of Einstein and Bohr also.

But let us return to a general scheme of global-theory creation. Why must the synthetic theories empirically supersede the reductionist ones?

The cross-breds' introduction creates contradictions in all the cross-theories and obliges them to accomodate to each other, to ,interpenetrate” and „enrich” each other. For instance, in the case considered ,the general theory of relativity and the quantum theory of fields, taken together in a new synthesis, have thus far enriched each other" (De Witt, 1980, p. 683). The enrichment revealed itself in the QFT methods' penetration into GR (the gravitational field quantization). From the other hand, much less-known the GR penetration into QFT manifested itself in discovering the so-called „Unruh effect” in flat spacetime.

A recent example of this, closely connected with the black hole evaporation process mentioned by Hawking, is the behaviour of accelerated particle detectors. Since in GR one often has to worry about the behaviour of accelerated observers, the behaviour of such detectors even in flat, Minkowski space-time is of interest. By building a simple model of a particle detector, and accelerating it in the vacuum state in flat space-time, it is possible to show that such a detector behaves exactly as if it were immersed in a thermal sea of particles of temperature $\mathrm{T}=8 \mathrm{Ilha} / \mathrm{kc}$ (Unruh, 1980, p. 154).

The $\mathrm{T}_{1}$ and $\mathrm{T}_{2}$ meeting results in that their domains of validity increase on $\mathrm{D}_{2}$ and $\mathrm{D}_{1}$ respectively: $\mathrm{T}_{1}$ 's domain expands for the sake of $\mathrm{D}_{2} \bullet \mathrm{T}_{2}$ 's domain increases owing to Dp The crosstheorie's domain of validity becomes equal to $2\left(D_{1}+D_{2}\right)$. For instance, the creation of photon theory (and of SR) on the road junction of classical mechanics, thermodynamics and electrodynamics was due to interpenetration of statistical mechanics and thermodynamics (Gibbs's and Einstein's investigations in the field of ,statistical thermodynamics”), of statistical mechanics and electrodynamics (Einstein's density-of-radiation fluctuation theory), of thermodynamics and electrodynamics (Planck's introduction of notions of temperature and entropy of radiation), of mechanics and electrodynamics (relativity principle, corpuscular theory of light). The development of Einstein's ideas by De Broglie (Pais, 1980) brought to endowing of ,ponderous matter” with wave properties, etc. 
The cross-bred objects can be compared with channels through which the ideas and methods of cross-theories interpenetrate leading to mutual changes in their contents. If we compare now synthetic theories with the reductionist ones, which forbid the existence of cross-breds, we can conclude the following. Even in the most favourable for the reduction case when the fundamental problems are solved already, the global theory validity domain increases due to $\mathrm{D} 2$ addition to $\mathrm{D}_{1}$. The fundamental theory $\mathrm{T}_{1}$, ,penetrates" into the phenomenological theory $\mathrm{T}_{2}$, but the reverse process of $T_{2}$ penetrating into $T_{1}$ is forbidden. That is why the domain of validity of synthetic ideal is two times larger than that of reductionist one. Namely that should provide of the synthetic programme over the reductionist ones.

Indeed, the cross-bred objects appearance leads to contradictions in each crosstheory. For instance, the introduction of photons contradicted classical radiation theory that explained interference and diffraction of light waves.

This way of looking at the problem showed in a drastic and direct way that a type of immediate reality has to be ascribed to Planck's quanta, that radiation must, therefore, possess a kind of molecular structure in energy, which of course contradicts Maxwell's theory. (Einstein, 1949:51).

But the contradictions exist only some definite period of time, since the crossbreds' occurence causes such changes in cross-theories, that in the long run resolve the contradictions. It leads to creation of new theories, that contain the old ones in transformed forms. The contradictions play the role of new theories creation „driving force".

Reflections of this type made it clear to me as long ago as shortly after 1900, i.e. shortly after Planck's trailblazing work, that neither mechanics nor electrodynamics could (except in limiting cases) claim exact validity. (Einstein, 1949:51).

In the case of a clash between GR and QFT the methodological scheme outlined can better be illustrated by the following example:

It is ironic that just at the time when the experimental tests are becoming possible, are being performed, and are verifying the prediction of the theory, the most determined assault on structure of the theory itself is taking place. In the attempt to make general relativity compatible with quantum field theory, many feel that the theory must be altered in some fashion. The most notable example of this is, of course, supergravity, with its addition of the gravitino. (Unruh, 1980:153).

The very revelation of the cross-contradiction is a problem to be solved out of the petty frameworks of special, concrete investigations. The cross-theory creation is a necessary stage. To reveal the cross-contradiction we need 'Gedankenexperiments', and not the real ones, with several-theories' abstract objects. But the most conservative part of the physical community consists of experimentalists. They can deal only with usual contradictions between theoretical predictions and experimental results. Yet not any anomaly indicates

the existence of cross-contradiction. Only some of them can create the problem situation. What are these anomalies? 
To answer the question we must consider the connection of cross-contradiction ocurrence with the occurence of anomalies. As we have already pointed out, the derivative systems contain invariant content of the empirical schemes, and the basic system contains invariant content of derivative ones. The crossbred system belongs to subsystems of derivative objects of all the fundamental theories $T_{1}, T_{2}, T_{3}, \ldots$ Hence the occurence of mutually-contradicting propositions in cross-bred theory is equivalent to occurence of contradictions in each $\mathrm{T}_{1}, \mathrm{~T}_{2}, \mathrm{~T}_{3}, \ldots$ And the latter is equivalent to installation of new relations between the derivative objects that are inconsistent with the old ones. But each derivative system is an invariant content of a certain set of empirical schemes. Hence the cross-contradiction leads not only to the contradictions between the crossbred propositions and a single experiment, but to the contradiction with the results of the whole set of experiments.

For example, the cross-contradiction between classical mechanics and classical electrodynamics in the crossbred theory - the planetary theory of atom - is equivalent to installation providing electrodynamics objects by new properties, inconsistent with the old ones. It leads to the following. The crossbred theory contradicts not only the experiments with some peculiar types of atoms, metals, for instance. It contradicts to experiments with all the atoms of Mendeleev's periodic table. According to cross-bred predictions, all the atoms should be unstable.

Thus, the cross-contradiction manifests itself (,appears") not in common deflections of cross-bred predictions with experimental results. Any fundamental theory is always surrounded by anomalies. The problem situation, created by the cross-contradiction, is characterized by the anomalies, that are out of the domain of usual deflections of theoretical predictions from experimental results. The attention of the researchers is directed on namely these ,,anomalous” anomalies, and a period of ,crisis” begins. This period was carefully described by T. S. Kuhn with a help of large number of history-of-science data. These data contain the transitions from geocentric astronomy to heliocentric one (,the state of Ptolemaic astronomy was a scandal before Copernicus's announcement" —Kuhn, 1957, pp. 138 - 140, 270271), from physics of Aristotle to Newtonian mechanics (,both Galileo's and Newton's contributions to the study of motion were initially focused upon difficulties discovered in ancient and medieval theory" - Kuhn, 1978, p. 206; 1957, pp. 237-260). It includes the transitions from the corpuscular theory of light to the wave theory that ,was announced in the midst of growing concern about anomalies in the relation of diffraction and polarization in Newton's theory" (Kuhn, 1977 , p. 206; 1958, pp. 27-45), from the phlogiston chemistry to oxygene one („Lavoisier's new chemistry was born after the observation of anomalous weight relations in combustion" - Kuhn, 1977, pp. 
206-207). The same material includes the transitions from the classical mechanics to the relativistic one (Kuhn, 1980), from the classical to the quantum mechanics that was born „from a variety of difficulties surrounding black-body radiation, specific heat, and the photoelectric effect" (Kuhn, 1977, p. 208; 1978). The crisis begins with the occurence of such anomalies that

...either because they are particularly striking or because they are educed repeatedly in many different laboratories, cannot be indefinitely ignored. Though they remain unassimilated, they impinge with gradually increasing force upon the consciousness of the scientific community.

As this process continues, the pattern of the community's gradually changes. At first, reports of unassimilated observations appear more and more frequently in the pages of laboratory notebooks or as asides in published reports. Then more and more research is turned to the anomaly itself. Those who are attempting to make it lawlike will increasingly quarrel over the meaning of the concepts and theories which they have long held in common without awareness or ambiguity. A few of them will begin critically to analyze the fabric of belief that has brought the community to its present impass. (Kuhn, 1977:262).

I think that in his works T. S. Kuhn correctly points out the necessity of a crisis as a preliminary stage of paradigm change. The model proposed not only can theoretically reproduce the necessity of crisises, but can also explain some important historical examples of Kuhn's 1977 paper The Function for Thought Experiments. For instance, Kuhn describes the Aristotle-galileo transition and finds Galileo's 'Gedankenexperiments' very important.

The concepts that Aristotle applied to the study of motion were, in some part, self-contradictory, and the contradiction was not entirely eliminated during the Middle Ages. Galileo's thought experiment brought the difficulty to the fore by confronting readers with the paradox implicit in their mode of thought [...] Similarly, Aristotle's concept of speed, with its two simultaneous criteria, can be applied without difficulty to most of the motions we see about us. Problems arise only for that class of motions, again rather rare, in which the criterion of instantaneous velocity and the criterion of average velocity lead to contradictory responses in qualitative applications. In both these cases the concepts are contradictory only in the sense that the individual who employs them runs the risk of self-contradiction. He may, that is, find himself in a situation where he can be forced to give incompatible answers to one and the same question. (Kuhn, 1977:251).

To sum up: the fundamental law can be refuted by the empirical evidence connected with other fundamental laws that contradict the first one.

\section{REFERENCES}

Birrell, N. D., Davies, P. C. W. (1982), Quantum Fields in Curved in Space, CUP.

Cartwright, N. (1983), How the Laws of Physics Lie, Oxford, Clarendon Press.

Chyba, C., Kaluza-Klein, F., Unified Field Theory and Apparent Four-dimensional space-time, American Journal of Physics, vol. 53(9), pp. 863-872.

De Witt, B. S. (1975), Quantum-Field Theory in Curved Spacetimes, Physics Reports, C. 19, pp. 297 -357.

De Witt, B. S. (1980), Quantum Gravity: the New Synthesis, [in:] Quantum Gravity 2, Oxford, pp. 680-745. Dirac, P. A. M. (1973), Development of the Physicist's Conception of Nature, [in:] Mehra J. (ed.), The

Physicist's Conception of Nature, D. Reidel, pp. 1-14. 
Einstein, A. (1949), Autobiographical Notes, [in:] Albert Einstein: Philosopher-Scientist (ed. by P. A. Schlipp), Evanston, Illinois, pp. 3-95.

Fulling, S. A. (1973), Nonuniqueness of Canonical Field Quantization in Riemannian Space-Time, The Physical Review, vol. 7, pp. 2850-2861.

Kuhn, T. S. (1957), The Copernican Revolution: Planetary Astronomy in the Development of Western Thought, Cambridge: Harvard University Press.

Kuhn, T. S. (1958), Newton's Optical Papers, [in:] Isaac Newton's Papers and Letters on Natural Philosophy, ed. by I. B. Cohen, Cambridge, MA, pp. 27-45.

Kuhn, T. S. (1977), Objectivity, Value Judgement and Theory Choice, [in:] The Essential Tension, University of Chicago Press, pp. 320-329.

Kuhn, T. S. (1977), A Function for Thought Experiments, [in:] The Essential Tension, University of Chicago Press, pp. 240-265.

Kuhn, T. S. (1978), Black-Body Theory and the Quantum Discontinuity, 1894-1912, Oxford and New York.

Kuhn, T. S. (1980), 77ie Halt and the Blind: Philosophy and History of Science, The British Journal for the Philosophy of Science, vol. 31, pp. 181-192.

Nugayev, R. M. (1987), The Genesis and Structure of Models in the Modern Theory of Gravity, International Studies in the Philosophy of Science, vol. 2, n 1, pp. 84-104.

Pais, A. (1980), Einstein on Particles, Fields, and the Quantum Theory, [in:] Some Strangeness in the Proportion, N. Y., pp. 197-251.

Podgoretzky, M. I., Smorodinsky, J. A. (1980), On the Axiomatic Structure of Physical Theories, [in:] The Physical Theory, Moscow, Nauka, pp. 53-61 (in Russian).

Stjopin, V. S. (1976), The Formation of a Scientific Theory, Minsk, Belorussian University Press (in Russian).

Unruh, W. G. (1980), Comment on Hawking's Paper, [in:] Some Strangeness in the Proportion, N. Y., pp. $153-155$ 